\title{
Prediction of solar active longitudes
}

\author{
L. Y. Zhang, H. N. Wang, and Z. L. Du
}

The National Astronomical Observatories, the Chinese Academy of Sciences, A20, Datun Rd., ChaoYang District, Beijing, PR China e-mail: zhangliyun@bao.ac.cn

Received 27 January 2008 / Accepted 1 April 2008

\section{ABSTRACT}

\begin{abstract}
Context. After considering the solar surface differential rotation, it was found that two persistent active longitudes of sunspots and of solar X-ray flares, separated by about 180 degrees, exist on the sun.

Aims. According to the migration rule of the active longitudes on the Sun, we predict the central positions of the solar active longitudes in Carrington frame.

Methods. The migration, $M$, of an active longitude on the $k$ th day of the $i$ th Carrington rotation in Carrington frame can be described as $M=T_{\mathrm{c}} \sum_{i=N_{0}}^{N_{i-1}}\left(\Omega_{i}-\Omega_{\mathrm{c}}\right)+k\left(\Omega_{i}-\Omega_{\mathrm{c}}\right)$, where $\Omega_{i}$ (sidereal) is the angular velocity of the active longitude in the $i$ th Carrington rotation, $\Omega_{\mathrm{c}}$ (sidereal), the angular velocity of Carrington frame, and $T_{\mathrm{c}}$, the Carrington rotation period. Solar active longitudes rotate in agreement with solar surface differential rotation law, which can be simply described as $\Omega_{i}=\Omega_{0}-B \sin ^{2}\left\langle\phi_{i}\right\rangle$, where $\Omega_{0}$ is the equatorial angular velocity, $B$ the differential rotation rate, and $\phi_{i}$, the mean latitude of solar activity in the $i$ th rotation. Parameters, $\Omega_{0}$ and $B$, can be obtained from observations. If the latitude in the $(i+1)$ th rotation is approximately that of the $i$ th rotation, the expected angular velocity of an active longitude in the $(i+1)$ th rotation can be described as $\Omega_{(i+1) p}=\Omega_{0}-B \sin ^{2}\left\langle\phi_{i}\right\rangle$. The central positions of active longitude, $\Lambda_{(i+1) k p}$, can be expected as well.

Results. We predict active longitudes of solar X-ray flares qualitatively between 1997-2006. Active longitudes with half width of $20^{\circ}-30^{\circ}$ cover $80 \%$ of $\mathrm{C}$-flares during the minimum of solar activities and X-flares during the maximum.

Conclusions. Solar active longitudes can be predicted by differential rotation parameters and latitude of solar activity.
\end{abstract}

Key words. Sun: activity - Sun: magnetic fields

\section{Introduction}

Currently, predictions of solar activities are mainly based on the typical properties of active regions, such as sunspot configuration, the complexity of magnetic fields, etc. This means that a short-term forecast can be made only after the appearance of an active region. Moreover, a flare can only be predicted qualitatively, without any information of its position, which is as important as quality (or magnitude) information, especially for a powerful flare. Svestka (1968) has ever tried to predict the dangerous longitudes of proton flares. But his result was not promising after he found the large extension of heliographic longitudes, which were occupied by active regions suspected of a proton-flare activity, particularly in the maximum phase of solar activity. In the following decades, the longitude prediction of solar activities has not been widely studied. However, through analyzing $\mathrm{X}$-ray flares data during the last three solar cycles, it has been found that active longitudes do not extend to a large scale in the Carrington frame and that they migrate in agreement with solar surface differential rotation, which makes longitude prediction possible according to the migration rule of active longitudes.

The subject of solar active longitudes has been studied for a long time. Although they have been found in various solar activity indices, such as sunspots (Bumber \& Howard 1969; Balthasar \& Schussler 1984), heliospheric magnetic fields (Ruzmaikin et al. 2001; Takalo \& Mursula 2002; Mursula \& Hiltula 2004), and flares (Bai \& Cliver 1990), they rotate with a variable velocity within a solar cycle (Balthasar et al. 1986), which results in their disappearance for long timescales in the Carrington reference frame. Recently it was found, by removing the migration in longitude resulting from solar surface differential rotation (Usoskin et al. 2005), that two persistent active longitudes of sunspots, separated by about $180^{\circ}$, have existed on the sun for more than 120 years. Despite a suspicion of persistent active longitudes by Pelt et al. (2005, 2006), who demonstrated that current evidence is not sufficient, these authors agree with the persistence of active longitudes over short times. Applying the same method of analysis to the data of solar X-ray flares, two persistent active zones were proposed for any class of flares during the last three solar cycles (Zhang et al. 2007a,b). Since persistent active longitudes have been proven to exist on the Sun, and their migrations in longitude are in agreement with solar surface differential rotation (Berdyugina \& Usoskin 2003; Bumba et al. 2000), we can predict their longitudinal positions, according to differential rotation parameters and the mean latitude of solar activity. We give for the first time a prediction of active longitudes, at least for a Carrington rotation in advance.

We first describe the method of obtaining differential parameters in Sect. 2, and then we present a prediction technique and results in Sect. 3. Finally, some conclusions and discussions are shown in Sect. 4.

\section{Differential rotation parameters}

As it is known that two active longitudes simultaneously appear $180^{\circ}$ apart in longitude (Warwick 1965; Svestka 1968), their central positions at the beginning of the data set in Carrington frame can be assumed as $\Lambda_{01}$ and $\Lambda_{02}$, which satisfy the following relation,

$\left|\Lambda_{01}-\Lambda_{02}\right|=180^{\circ}$. 
Solar surface differential rotation law is described simply as,

$\Omega=\Omega_{0}-B \sin ^{2}\langle\phi\rangle$,

where $\Omega$ (deg/day) is the angular velocity at a given latitude $\phi$, while $\Omega_{0}$ (deg/day) is the equatorial angular velocity and $B$ (deg/day) describes differential rotation rate. Parameters $\Omega_{0}$ (sidereal) and $B$ need to be determined from observations. If the time step is taken as the Carrington rotation period, then Eq. (1) takes the form

$\Omega_{i}=\Omega_{0}-B \sin ^{2}\left\langle\phi_{i}\right\rangle$,

where $\Omega_{i}$ (deg/day, sidereal) is the angular velocity of either of two active longitudes in the $i$ th Carrington rotation, index, $i$, denotes the $i$ th Carrington rotation, and $\phi_{i}$ is the peak-intensity weighted average latitude of $\mathrm{X}$-ray flares during this Carrington rotation (Zhang et al. 2007b). For Carrington rotations with no flare activity, the linear interpolation of mean latitude is used.

With the arrival of the $k$ th day in the $i$ th Carrington rotation, the migration, $M$, of an active longitude in the Carrington reference frame is determined, according to the solar surface differential rotation law, by

$M=T_{\mathrm{c}} \sum_{i=N_{0}}^{N_{i-1}}\left(\Omega_{i}-\Omega_{\mathrm{c}}\right)+k\left(\Omega_{i}-\Omega_{\mathrm{c}}\right)$,

where $N_{0}$ and $N_{i-1}$ denote the numbers of the beginning Carrington rotation and the $(i-1)$ th rotation in the data set, $T_{\mathrm{c}}$, the Carrington rotation period (27.27 days), and $\Omega_{\mathrm{c}}$, the sidereal angular velocity of Carrington frame (14.1844 deg/day). The migration here is calculated more accurately than the migration in Zhang et al. (2007b), which makes the obtained parameters more close to the real ones.

Considering the migration, $M$, the central positions of the two active longitudes in Carrington frame can be denoted as $\Lambda_{k i 1}$ and $\Lambda_{k i 2}$,

$\Lambda_{i k 1}=\left(\Lambda_{01}+M\right) \operatorname{Mod} 360^{\circ}$,

$\Lambda_{i k 2}=\left(\Lambda_{02}+M\right) \operatorname{Mod} 360^{\circ}$,

where $n \operatorname{Mod} 360^{\circ}$ means the $n$ modulo $360^{\circ}$ within the range $\left[0^{\circ}, 360^{\circ}\right]$, e.g., $-10^{\circ} \operatorname{Mod} 360^{\circ}$ is $350^{\circ}$.

The deviation between the longitudinal position of a flare, $\lambda_{i k}$, which occurred in the $k$ th day in the $i$ th rotation, and the center of an active longitude band, $\Lambda_{i k 1}$ or $\Lambda_{i k 2}$, is defined as

$\Delta_{i k}=\min \left(\left|\lambda_{i k}-\Lambda_{i k 1}\right|,\left|\lambda_{i k}-\Lambda_{i k 2}\right|\right)$,

where $k$ is fractional, e.g., $k$ is 0.50 for the flare occurred at 12:00 UT in the first day in a rotation, and 1.50 at 12:00 UT in the second day. The difference $|\lambda-\Lambda|$ is circular. Formally, if $\lambda=1$ and $\Lambda=359,|\lambda-\Lambda|=358$, while for the circular distance, it is 2 .

The mean discrepancy between the model and the data is then defined as

$\epsilon=\frac{1}{N} \sum_{i} \sum_{k} \Delta_{i k}^{2}$

where $N$ is the total number of flares in the data set. Varying the value of $\Omega_{0}$ in the interval $[13.50,15.0]$ with step $=0.01 ; B$ in $[1.0,4.0]$ with step of 0.01 ; and $\Lambda_{01}$ in $\left[0^{\circ}, 360^{\circ}\right]$ with step of $1^{\circ}$, we use the data obtained between 1977-1996 to search for a group of $\Omega_{0}, B$, and $\Lambda_{01}$, which minimizes the discrepancy $\epsilon$. Then, the active longitudes in Carrington frame, $\Lambda_{i k 1}$ and $\Lambda_{i k 2}$, from 1977 to 1996 can be determined by the best fit parameters.

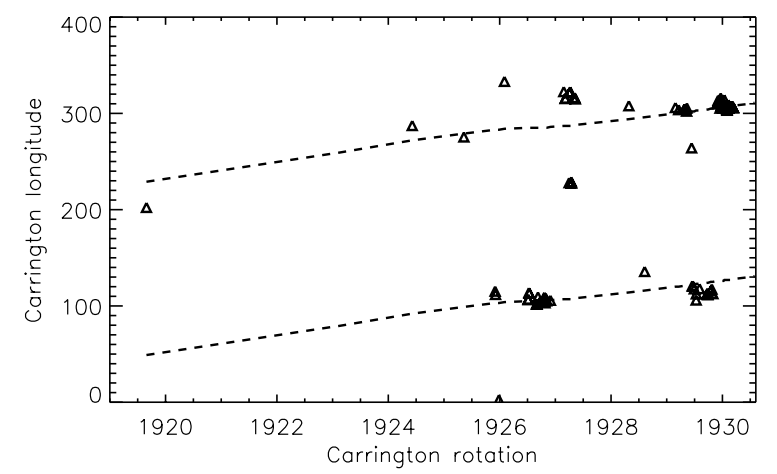

Fig. 1. A simulated prediction of the active longitudes in 1997 in the northern hemisphere. The horizontal axis denotes Carrington rotation number and the vertical axis denotes the Carrington longitude. The two lines denote the predicted centers of the two active longitudes in 1997 in northern hemisphere, while triangles, " $\triangle$ "s, the observed X-ray flares.

\section{Prediction on active longitudes}

Parameters, $\Omega_{0}$ and $B$, derived from the observations can be extrapolated for the subsequent rotation. For example, the parameters derived from the observations during the period from the first rotation to the $i$ th, can be used as the first estimate for the $(i+1)$ th rotation. The latitude in the $(i+1)$ th rotation is approximately the mean latitude of solar activity in the $i$ th rotation, $\phi_{i}$. Then, the expected angular velocity of active longitudes in the $(i+1)$ th rotation, $\Omega_{(i+1) p}$, can be given by,

$\Omega_{(i+1) p}=\Omega_{0}-B \sin ^{2}\left\langle\phi_{i}\right\rangle$,

where the subindex, $p$, stands for prediction. Predicted active longitudes in the $k$ th day in the $(i+1)$ th rotation, $\Lambda_{(i+1) k 1 p}$ and $\Lambda_{(i+1) k 2 p}$, can be described as the following expressions:

$\Lambda_{(i+1) k 1 p}=\left\{\Lambda_{i 1}+k\left(\Omega_{(i+1) p}-\Omega_{\mathrm{c}}\right)\right\} \operatorname{Mod} 360^{\circ}$,

$\Lambda_{(i+1) k 2 p}=\left\{\Lambda_{i 2}+k\left(\Omega_{(i+1) p}-\Omega_{\mathrm{c}}\right)\right\} \operatorname{Mod} 360^{\circ}$,

where $\Lambda_{i 1}$ and $\Lambda_{i 2}$ denote longitudinal coordinates of active zones in Carrington frame at the end of the $i$ th rotation. We can predict active longitudes in Carrington frame at any time in the $(i+1)$ th rotation when $k$ is taken over the Carrington period, $0-27.27$ days.

At the end of the $(i+1)$ th Carrington rotation, we search for the best fit parameters again, using the observations from the beginning up to this rotation. We can also calculate the mean latitude of flares in the $(i+1)$ th rotation, $\phi_{i+1}$. Then, we can predict the active longitudes in the $(i+2)$ th rotation. Repeating the method from the first Carrington rotation in 1997 to the end of 2006, we obtain a simulated prediction for these ten years.

As an example, a simulated prediction of active longitudes in 1997 is shown in Fig. 1. The two dashed lines represent the predicted centers of active longitudes. The triangles, $\Delta$, denote the distribution of the observed longitudes of X-ray flares. From this figure, it can be seen that the first flare in this year occurred very close to the predicted active longitude. Despite the absence of flares during several rotations in early 1997, most of the observed flares occurred close to the predicted active longitudes.

To characterize the predicted results and estimate their significance, we introduce a measurement of concentration. The half width of the longitude band, $w_{\mathrm{h}}$, is defined as the distance from the predicted active longitudes (the dashed lines in Fig. 2) to a certain extension (the dotted lines in Fig. 2, parallel to the 


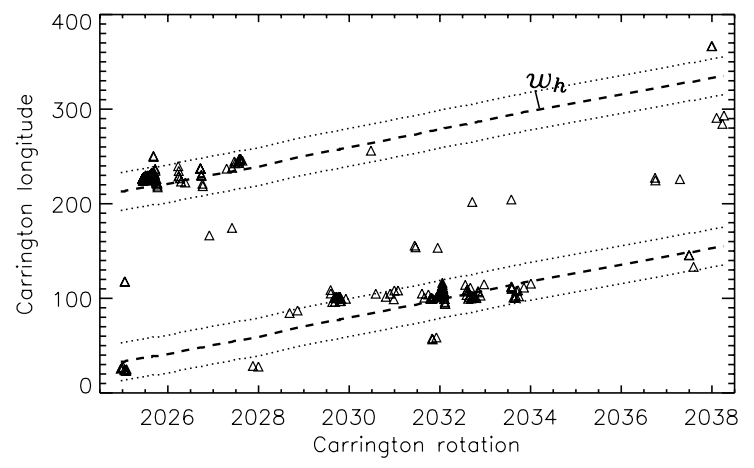

Fig. 2. A simulated prediction of the central active longitudes in 2005 in the northern hemisphere. The horizontal axis denotes Carrington rotation number and the vertical axis denotes the Carrington longitude. The two dashed lines denote the centers of the predicted active longitudes, and $w_{\mathrm{h}}$ is the distance between the dashed line and dotted lines, " $\triangle$ "s, the observed X-ray flares.

dashed line). The number of flares occurred in the two longitude bands, $N_{\mathrm{f}}$, is obtained by,

$N_{\mathrm{f}}=\sum_{i, k} 1$, if $\left|\lambda_{i k}-\Lambda_{i k 1 p}\right|<w_{\mathrm{h}}$ or $\left|\lambda_{i k}-\Lambda_{i k 2 p}\right|<w_{\mathrm{h}}$.

The confidence level of the flares occurring in the longitude bands is given by the probability, $p$,

$p=N_{\mathrm{f}} / N_{\mathrm{t}}$,

where $N_{\mathrm{t}}$ is the total number of all X-ray flares. Varying the value $w_{\mathrm{h}}$ from $0^{\circ}$ to $90^{\circ}$ with step of $1^{\circ}$, we obtain the distribution of the probability, $p$, versus the half width of longitude band, $w_{\mathrm{h}}$, as shown in Fig. 3 (for the years 2000 and 2005, as examples). The confidence level, $p$, corresponds to values from 0 to 1 , where 0 corresponds to $w_{\mathrm{h}}$ of $0^{\circ}$ and 1 to $w_{\mathrm{h}}$ of $90^{\circ}$.

We consider $w_{\mathrm{h}}$ as valid half width $(V H W)$ of active longitudes, which include $80 \%$ of the flares as shown in Fig. 3 (horizontal dash-dotted line). The statistical confidence levels of 0.68 and 0.90 customary in scientific usage are also shown there for comparison. The two solid curves denote $p$ versus $w_{\mathrm{h}}$ distribution for C-flares in 2005 and 2000, and the dashed line for $\mathrm{X}$-flares in 2000 . To see the asymmetric distribution of flare occurrence clearly, we also show the confidence level corresponding to the assumed uniform distribution (the inclining dotted line). From this figure, we can see that the probability of 0.68 corresponds to the half width of $10^{\circ}$ for X-flares in 2000 (during solar maximum) and for C-flares in 2005 (during solar minimum). This half width is narrow enough to be used in solar active longitude prediction, even though the probability is not very great. If we take 90 percent as a criterion, the half width increases to nearly $50^{\circ}$ for X-flares, which results in a less larger extension. Taking both aspects (great probability and small extension) into account, the probability of $80 \%$ is taken as a criteria and the corresponding half width of the active longitudes is considered as the VHW.

On the one hand, from Fig. 3. one can see that VHW of the longitude bands for C-flares during solar maximum (C-2000) is broader than that during minimum (C-2005). When the half width is $17^{\circ}$, the confidence level of flare occurrence increases to 0.80 in 2005 . While the same confidence level $(0.80)$ corresponds to a $w_{\mathrm{h}}$ of $64^{\circ}$ in 2000 . The VHW during solar maximum is about $50^{\circ}$ broader than the VHW during solar minimum for $\mathrm{C}$-flares. This may be the reason why preferred longitudes were often found over short timescales, especially near the minimum

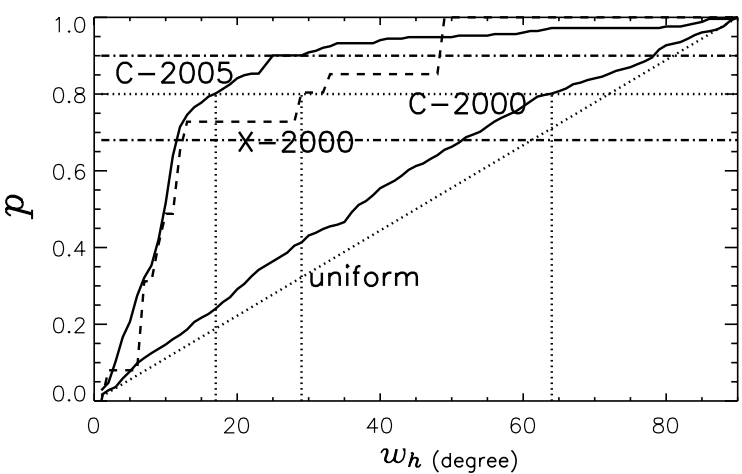

Fig. 3. Comparison among the half widths of longitude bands in different time periods. The $w_{\mathrm{h}}$ denotes the half width of the predicted longitude bands, and the $p$ the confidence level of flares occurring in the longitude bands. The two solid curves denote the distribution for C-flares in 2005 and 2000, the dashed line for X-flares in 2000, and the dotted line for the uniform distribution. The horizontal dotted line corresponds to the confidence level of 0.80 . The two horizontal dash-dotted lines correspond to the confidence levels of 0.68 and 0.90 .

of a solar cycle (Vitinskij 1969, 1990; Bai 1987; Ozguc \& Altas 1996; Benevolenskaya et al. 1999; Bumba et al. 2000; Usoskin et al. 2007).

On the other hand, the VHW for X-flares during solar maximum is only about $30^{\circ}$. Because there were only $10 \mathrm{X}$-flares in the northern hemisphere in 2000, the distribution line is not smooth. The confidence level is up to $80 \%$ at the $w_{\mathrm{h}}$ of $29^{\circ}$, which implies that active longitudes, with valid half width of $20^{\circ}-30^{\circ}$, exist during the entire solar cycle. Active longitudes can be found through $\mathrm{C}$-flares during solar minimum and through X-flares during the maximum. This is very important in estimating the geo-space environment.

The prediction results from the whole solar cycle 23 are shown in Fig. 4. The solid line denotes the probability of C-flares, the dashed line shows the X-flares, and the inclining dotted line indicates the uniform distribution. From this figure, we note that if we take the same $w_{\mathrm{h}}$ of the two classes of flares, e.g., $45^{\circ}$, the probability of X-flare occurrence in the predicted longitude bands, 0.81 , is much higher than that of C-flares (0.63), which is much higher than that of uniform distribution (0.50). These confidence levels correspond to the magnitude of the non-axisymmetry $\Gamma_{\mathrm{n}}$ defined by Zhang et al. (2007b), 0.61, for X-flares, and 0.26 for C-flares, which confirms the conclusion that solar activities do occur around double preferred longitudes in each hemisphere of the Sun for decades and that non-axisymmetry will increase with the importance of events. The statistical confidence level $0.68(0.90)$ corresponds to the half width of $28^{\circ}\left(61^{\circ}\right)$ for X-flares and $50^{\circ}\left(75^{\circ}\right)$ for C-flares. The VHW of X-flares $\left(42^{\circ}\right)$ is about $20^{\circ}$ narrower than that of $\mathrm{C}$-flares $\left(64^{\circ}\right)$, which indicates that the preferred longitudes of large-scale active regions or major flares were easily found (Bumba \& Howard 1965; Dodson \& Hedeman 1975; Gaizauskas et al. 1983; Bumba \& Obridko 1969), even for long timescales (Bai 1988; Jetsu 1997). The greater confidence level in a smaller longitudinal extension for X-flares compared to C-flares increases the importance of the active longitude prediction.

\section{Conclusions and discussions}

Based on the surface differential rotation model of the Sun, we construct a method for predicting the centers of solar active longitudes in the Carrington frame. After analyzing the raw data of 


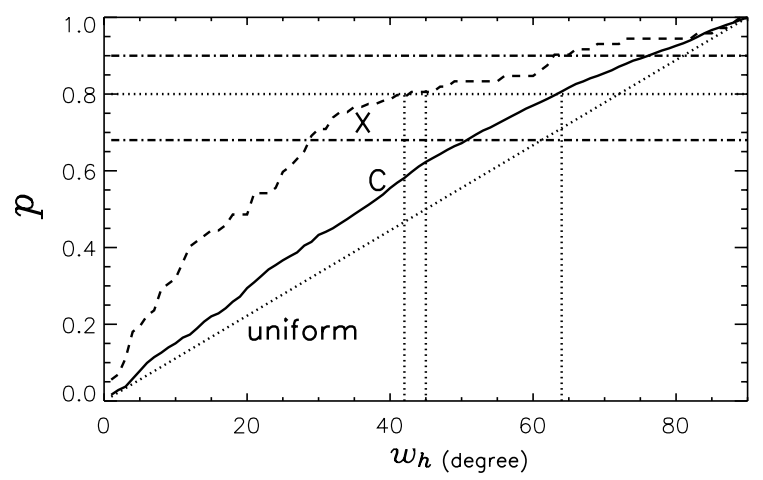

Fig. 4. The distributions of $p$ versus $w_{\mathrm{h}}$ of C-flares (solid), X-flares (dashed), and uniform distribution (dash-dotted) for solar cycle 23. The horizontal dotted line corresponds to the confidence level of 0.80 . The two horizontal dash-dotted lines correspond to the confidence levels of 0.68 and 0.90 .

X-ray flares (402 X-flares and 28004 C-flares) and predicting active longitudes for solar cycle 23 , we obtained the following conclusions:

1. Solar active longitudes can be predicted by the differential rotation parameters and the mean latitude of solar activities in a Carrington rotation.

2. Active longitudes with valid half width of $20^{\circ}-30^{\circ}$ exist during the entire solar cycle. These longitudes can be found through C-flares during solar minimum and through X-flares during the maximum. This is very important for geo-space environment research and space weather prediction.

Applying this method, one can predict potentially dangerous longitudes at least one solar rotation in advance. Especially, during the period around solar minimum, e.g., 1997, possible active longitudes can be proposed a year in advance because of the absence of activities and the unchanging of the prediction parameters. Even during the maximum, centers of active zones can be estimated several rotations in advance. But the prediction is modified according to the observation. In reality, not all the solar activity phenomena can result in strong geo-space effects. Only those which appear at specific locations, e.g., close to the center of the west limb of the solar disk, are potentially geo-effective. Therefore, we can estimate the geo-space environment according to the predicted dangerous longitude and the heliographic site during a certain time period. This is only the primary work on the prediction of solar active longitudes. The following research will focus on improving the prediction validity.

Because of the south-north asymmetry (Temmer 2006; Waldmeier 1971; Verma 1993), we analyze the data in each hemisphere separately. The above results are derived from the northern hemisphere. The predicted results in the southern hemisphere, not shown here, are similar to those in the northern. But the rotating velocity in the southern hemisphere is found to be slower than that in the northern, which results in faster migration of the active zones in the northern hemisphere than in the southern hemisphere by about $100 \mathrm{deg} /$ year. To compare migrations of the active zones conveniently in both hemispheres, the shifts of an active zone in phase $\left(\Lambda / 360^{\circ}\right)$ in each hemisphere during solar cycle 22 are shown in Fig. 5. From the upper panel, it can be seen that the phase of the active zone in the northern hemisphere in Carrington frame increases continuously during the whole solar cycle, which implies that the active zone constantly overtakes the Carrington meridian. The slow increase at the beginning of the cycle and more quickly at the end
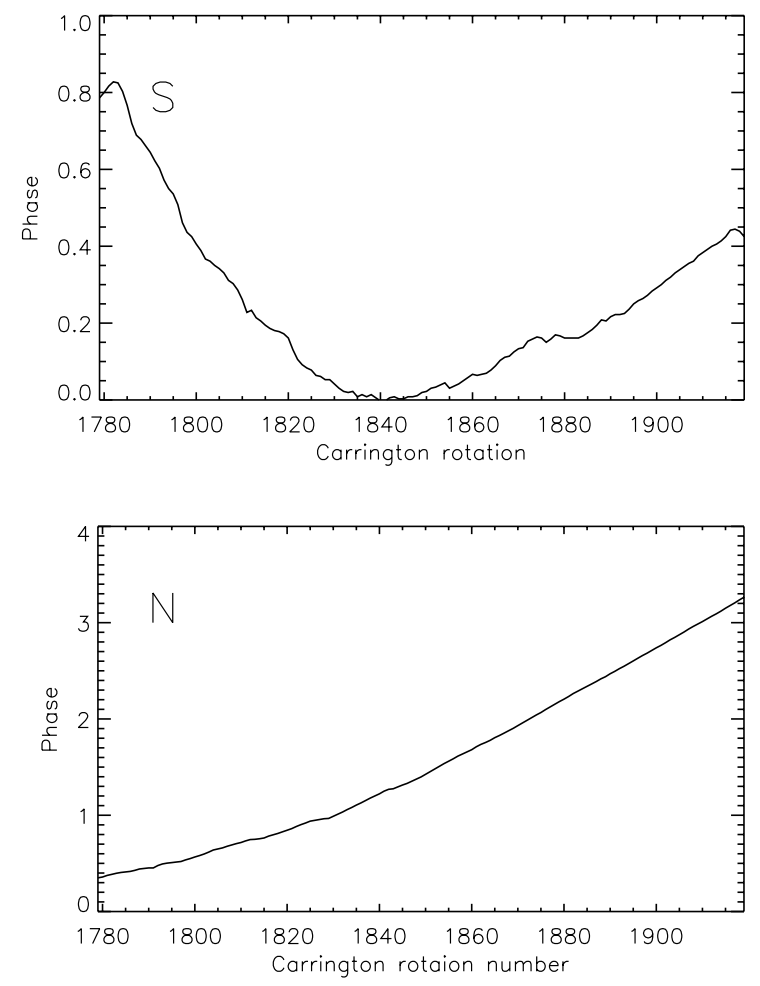

Fig. 5. Comparison of the phase shifts of the active longitudes in the two solar hemispheres in Carrington frame. The upper panel shows the northern hemisphere during solar cycle 22 and the lower panel shows the southern. The vertical axis denotes the phase of an active zone, the horizontal denotes the Carrington rotation number.

corresponds to the flares occurring at high and low latitudes at that time. The lower panel suggests that the active zone lags behind the Carrington meridian at the beginning of a solar cycle and overtakes it at the end, which is also in agreement with the latitude of solar activity at that time. In spite of the faster migration of the active zones in the northern hemisphere, their migration in each hemisphere can be determined by the solar differential rotation, respectively.

Although this inconsistent migration of active longitudes in the two hemispheres derived from flares is in agreement with those derived from sunspots (Berdyugina et al. 2006), the active longitudes determined by solar X-ray flares seem to rotate faster than those determined by sunspots (Usoskin et al. 2005). This difference is probably caused by the different effects of the solar differential rotation model on the depth where flares occur and where sunspots appear. Another probable reason might be that the latitudes of flares are lower than those of sunspots for more sunspots than flares appear at high latitudes at the beginning of a solar cycle. This provides new constraints for the development of solar dynamo models, as was recently undertaken by Moss (1999, 2004), Fluri \& Berdyugina (2004), Jarvinen et al. (2005), and Jiang \& Wang (2007).

Acknowledgements. This work is supported by Chinese Academy of Sciences Projects KJCX2-YW-T04 and KGCX3-SYW-403-10, National Ministry of Science and Technology grant No. 2006CB806307, and National Natural Science Foundation of China (NSFC) grants 10673017 and 10733020 . We thank the referee for useful suggestions on improving this manuscript. We also thank the GOES and NGDC web teams for data. 


\section{References}

Bai, T. 1987, ApJ, 314, 795

Bai, T. 1988, ApJ, 328, 860

Bai, T., \& Cliver, E. W. 1990, ApJ, 363, 299

Balthasar, H., \& Schüssler, M. 1984, Sol. Phys., 93, 177

Balthasar, H., Vazquez, M., \& Woehl, H. 1986, A\&A, 155, 87

Benevolenskaya, E. E., Hoeksema, J. T., Kosovichev, A. G., \& Scherrer, P. H. 1999, ApJ, 517, L163

Berdyugina, S. V., \& Usoskin, I. G. 2003, A\&A, 405, 1121

Berdyugina, S. V., Moss, D., Sokoloff, D., \& Usoskin, I. G. 2006, A\&A, 445, 703

Bumba, V., \& Howard, R. 1965, ApJ, 141, 1492

Bumba, V., \& Obridko, V. N. 1969, Sol. Phys., 6, 104

Bumba, V., Garcia, A., \& Klvaňa, M. 2000, Sol. Phys., 196, 403

Dodson, H. W., \& Hedeman, E. R. 1975, Sol. Phys., 42, 121

Fluri, D. M., \& Berdyugina, S. V. 2004, Sol. Phys., 224, 153

Gaizauskas, V., Harvey, K. L., Harvey, J. W., \& Zwaan, C. 1983, ApJ, 265, 1056

Järvinen, S. P., Berdyugina, S. V., \& Strassmeier, K. G. 2005, A\&A, 440, 735

Jetsu, L., Pohjolainen, S., Pelt, J., \& Tuominen, I. 1997, A\&A, 318, 293

Jiang, J., \& Wang, J. X. 2007, MNRAS, 377, 711

Moss, D. 1999, MNRAS, 306, 300
Moss, D. 2004, MNRAS, 352, L17

Mursula, K., \& Hiltula, T. 2004, Sol. Phys., 224, 133

Özgüc, A., \& Altas, L. 1996, Sol. Phys., 163, 327

Pelt, J., Tuominen, I., \& Brooke, J. 2005, A\&A, 429, 1093

Pelt, J., Brooke, J. M., Korpi, M., \& Tuominen, I. 2006, A\&A, 460, 875

Ruzmaikin, A., Feynman, J., Neugebauer, M., \& Smith, E. J. 2001, J. Geophys. Res., 106, 8363

Švestka, Z. 1968, Sol. Phys., 4, 18

Takalo, J., \& Mursula, K. 2002, Geophys. Res. Lett., 29(9), 1317

Temmer, M., Rybák, J., Bendík, P., et al. 2006, A\&A, 447, 735

Usoskin, I. G., Berdyugina, S. V., \& Poutanen, J. 2005, A\&A, 441, 347

Usoskin, I. G., Berdyugina, S. V., Moss, D., \& Sokoloff, D. D. 2007, Adv. Space Res., 40, 951

Verma, V. K. 1993, ApJ, 403, 797

Vitinskij, Yu. I. 1969, Sol. Phys., 7, 210

Vitinskij, Yu. I. 1990, Soln. Dannye, Byull., 8/90, 88

Waldmeier, M. 1971, Sol. Phys., 20, 332

Warwick, C. S. 1965, ApJ, 141, 500

Zhang, L. Y., Cui, Y. M., Du, Z. L., \& Wang, H. N. 2007a, Adv. Space Res., 40, 970

Zhang, L. Y., Wang, H. N., Du, Z. L., Cui, Y. M., \& He, H. 2007b, A\&A, 471, 711 